\title{
Epigenetic high regulation of ATAD2 regulates the Hh pathway in human hepatocellular carcinoma
}

\author{
GANG WU, XIAOJUN LU, YAWEI WANG, HUI HE, XIANGYU MENG, \\ SHUGUAN XIA, KUNMING ZHEN and YONGFENG LIU \\ Department of General Surgery, The First Affiliated Hospital of China Medical University, \\ Shenyang, Liaoning 110001, P.R. China
}

Received February 13, 2014; Accepted April 17, 2014

DOI: $10.3892 /$ ijo.2014.2416

\begin{abstract}
ATAD2 is associated with many cellular progresses such as cell growth, differentiation and apoptosis. Some studies suggest ATAD2 is highly expressed in cancer cells. In our previous studies, we found that ATAD2 is highly expressed in HCC tissues, compared with adjacent normal tissues, and patients with high expression of ATAD2 had a poorer prognosis. Moreover, we found mir-372 can regulate the expression of ATAD2 in HCC cell lines. We also detected a relationship between the mRNA expression of ATAD2 and Ptch1 by gene microarray. Here, we completed the function studies of ATAD2 in vivo and in vitro, and tested whether ATAD2 could regulate the Hh pathway. ATAD2 and $\mathrm{Hh}$ pathway protein expressions in $80 \mathrm{HCC}$ specimens were examined by immunohistochemistry (IHC). The mRNA expression of ATAD2 and Hh pathway members in paired-HCC tissues and cell lines were, respectively, analyzed using quantitative PCR. ATAD2-RNAi was transduced into HCCLM3 and Huh7 cells, using a lentiviral vector. The effect of ATAD2 in HCC cell lines on cell cycle and apoptosis were evaluated by flow cytometry. Tumorigenicity experiments in nude mice were performed to test the function of ATAD2 in vivo. Pharmacological regulation of Hh signaling was performed to test the relation between the ATAD2 and Hh pathways and C-myc. We found that ATAD2 and Ptch1 were both highly expressed in HCC tissues, compared with paired normal hepatic tissues. In addition, we found that ATAD2 could affect the expression of the Hh pathway by PCR and western blot anaysis in HCC cell lines, by observing the outcome before and after transfection. We speculate that ATAD2 cooperates with the MYC gene to regulate the expression
\end{abstract}

Correspondence to: Professor Gang Wu, Department of General Surgery, The First Affiliated Hospital of China Medical University, Shenyang, Liaoning 110001, P.R. China

E-mail:wugzwl@gmail.com

Key words: ATAD2, patched homolog 1, Hedgehog pathway, hepatocellular carcinoma, c-Myc of SMO and Gli, activating the Hh pathway and inducing an active feedback of the Hh pathway.

\section{Introduction}

Hepatocellular carcinoma (HCC) is the fifth most common malignant tumor worldwide with an incidence of about 626,000 cases each year $(1,2)$. In China and Southeast Asia, HCC is highly associated with viral hepatitis B and cirrhosis (3). Prognosis of patients with HCC have been improved largely because the surgical techniques and diagnostic methods have greatly improved in recent years, but long-term prognosis is still unsatisfactory largely due to the high recurrence and invasion rates even after resection $(50-70 \%$ at five years) $(4,5)$. How to predict which patients are likely to experience recurrence earlier after resection is a challenge to surgeons.

ATAD2, a member of the AAA+ ATPase family of proteins, was identified by microarray analysis (6). ATAD2, the predicted protein product which contains both a bromodomain and an ATPase domain, maps to chromosome 8q24, in a region that is frequently found amplified in cancer (7). The structure of ATAD2 suggests its functions relate to genome regulation including cell proliferation, differentiation and apoptosis. Studies have revealed that ATAD2 is highly expressed in several types of tumors such as breast cancer, lung cancer, large $\beta$-cell lymphoma (8-10). It is also highly expressed in hepatocellular carcinoma detected by RNA-seq (11).

The sonic hedgehog ( $\mathrm{SHH})$ signaling pathway was shown to play a critical role in hepatocellular carcinoma, regulating the cancer cells growth and differentiation (12-14). $\mathrm{HH}$ pathway is frequently activated in $\mathrm{HCC}$, and patched homolog 1 (Ptch1) and glioma-associated oncogene-1 (like Gli2) are the key genes in the Hh pathway. Hh protein binds to its receptor human Ptch1, and relieves Ptch1 inhibition on smoothened (SMO), subsequently Smo triggers a series of intra-cellular events with resultant activation of the zinc finger transcription effectors, glioma-associated oncogenes (Gli1, Gli2, Gli3) transcription factors, which induce the expression of numerous target genes, such as Ptch1, Hip, Gli2 and Wnt, regulating proliferation, and differentiation (15-17).

In our previous studies, we detected ATAD2 as highly expressed in HCC tissues, compared with adjacent normal 
tissues, and the patients with high-expression ATAD2 had a poorer prognosis, and ATAD2 influenced HCC cells proliferation, invasion and migration by transfection with ATAD2-siRNA-plasmid (18). Moreover, we also found mir-372 can regulate the expression of ATAD2 in HCC cell lines, and ATAD2 is the target gene of mir-372. In the present study, firstly, we found that there was a relationship between the mRNA expression of ATAD 2 and PTCH1 by PCR microarray (Fig. 1). Then, we further investigated the function of ATAD2 in vivo and in vitro by transfection with ATAD2-RNAi-lentivirus, and tested whether ATAD2 could regulate the Hh pathway. Moreover we investigated whether ATAD2 could activate the Hh pathway in HCC.

\section{Materials and methods}

Human Signal Transduction PathwayFinder PCR Array. The Human Signal Transduction PathwayFinder RT ${ }^{2}$ Profiler PCR Array (SABiosciences) profiles the expression of 84 key genes responsive to signal transduction pathway activation or inhibition. We set the two groups as normal control, and ATAD2-RNAi groups. The GAPDH gene was used as a reference control. The cDNA normal control, and ATAD2-RNAi groups were inserted in PCR array. Following data collection, the relative levels of gene expression were presented as $\Delta \mathrm{Ct}=\mathrm{Ct}$ gene-Ct reference and the fold change in gene expression was calculated using the $2^{-\Delta \Delta C t}$ method.

Hepatic cancer patients and liver cancer cell lines. Hepatic cancer tissue and normal hepatic cancer were obtained from 80 HCC patients. All patients were operated in the First Affiliated Hospital of China Medical University between March, 2002 and December, 2008. Among them, 58 were men and 22 were women, with the median age of 56 years (range, 30 to 78.5 years). No patient had been pretreated with preoperative radiotherapy or chemotherapy before surgical resection. Up to December, 2008 (the censor date), histologic diagnosis and differentiation were evaluated independently by 3 pathologists using hematoxylin-stained and eosinstained slides according to the WHO classification system (4). The total of 80 fresh specimens including both tumor tissues and corresponding paired non-cancerous parenchyma were snap-frozen in liquid nitrogen and stored at $-80^{\circ} \mathrm{C}$ immediately after resection until processing. The project protocol was approved by the Institutional Ethics Committee of China Medical University before initiation of the study, and all patients provided a written informed consent for the use of the tumor tissues for clinical research. The liver cancer cell lines Huh7, HCCLM3 were obtained from Shanghai Cell Bank (Shanghai, China). The Huh7, HCCLM3 cell lines were cultured in DMEM (Gibco, USA).

RNA preparation and quantitative real-time PCR. Total RNA was extracted according to the manufacturer's instructions from approximately $100 \mathrm{mg}$ of tissue for each of the 40 paired samples and liver cancer cell lines using TRIzol reagent (Invitrogen). The ATAD2, PTCH1, Gli2, SMO and GADPH primers were purchased from Takara Company (Japan). The GAPDH gene was used as a reference control for ATAD2, PTCH1, Gli2 and SMO. The relative levels of gene expres- sion were represented as $\Delta \mathrm{Ct}=\mathrm{Ct}$ gene-Ct reference and the fold change in gene expression was calculated using the $2^{-\Delta \Delta C t}$ method. The primer sequences are: ATAD2 forward, GGA ATCCCAAACCACTGGACA and reverse, GGTAGCGT CGTCGTAAAGCACA; Gli2 forward, TGGCCGCTTCAGA TGACAGATGTTG and reverse CGTTAGCCGAATGTCAG CCGTGAAG; PTCH1 forward, ATCCATAATGTCTGGAA CTTTGCTG and reverse, CATGCTAGGTCGCCAATGGTA; SMO forward, TGCCAGCAAGATCAACGAGA and reverse, GCAGCTGAAGGTAATGAGCACAA; C-MYC forward, GCAGCTGCTTAGACGCTGGA and reverse CGCAGT AGAAATACGGCTGCAC.

Cell lysate preparation and western blot analysis. Cell lysates were prepared by using a homogenate buffer containing $20 \mathrm{mM}$ Tris, $100 \mathrm{mM} \mathrm{NaCl}, 0.1 \mathrm{mM}$ PMSF, $10 \mu \mathrm{g} / \mathrm{ml}$ aprotinin and $1 \%$ NP-40. After sonication and centrifugation $\left(12,000 \mathrm{rpm}\right.$ for $15 \mathrm{~min}$ at $\left.4^{\circ} \mathrm{C}\right)$, protein concentrations were measured with the Bradford method (Bio-Rad, Hercules, CA). SDS-polyacrylamide gel electrophoresis techniques were used to separate proteins ( $40 \mu \mathrm{g} / \mathrm{lane}$ ) on 4 to $15 \%$ linear gradient gel. Proteins were then transferred to polyvinylidene difluoride membranes (Immobilon-P, Millipore, Bedford, MA) by semidry electroblotting and probed with either rabbit monoclonal anti-human for human studies, or for control anti-glyceraldehyde-3-phosphate dehydrogenase (GAPDH; Amblion Inc, Woodward, TX, USA), primary antibodies were used. Bands were visualized using horseradish peroxidase-conjugated secondary antibodies goat anti-rabbit. The loading control for the human samples was performed with GAPDH (1:5,000; rabbit IgG, USA.) Equal levels of gel loading were confirmed by stripping blots and then reprobing with GAPDH antibodies, respectively. ATAD2 antibody was purchased from Abcam company, and PTCH1, SMO, Gli2 antibody purchased from Santa Cruz Biotechnology.

Immunohistochemistry. Surgical specimens were immediately stored at $-80^{\circ} \mathrm{C}$. Serial cryostat sections $(5-\mu \mathrm{m})$ were fixed with $10 \%$ formalin and embedded in paraffin. After general deparaffinization, antigen retrieval was carried out for $30 \mathrm{sec}$ with an autoclave using $0.01 \mathrm{~mol} / \mathrm{l}$ sodium citrate buffer, pH 6.0. $\mathrm{H}_{2} \mathrm{O}_{2}(0.3 \%)$ was used to block endogenous peroxidase activity for $30 \mathrm{~min}$ at $37^{\circ} \mathrm{C}$, and non-specific immunoglobulin binding sites were blocked by normal goat serum for $30 \mathrm{~min}$ at $37^{\circ} \mathrm{C}$. Sections were then incubated overnight with primary antibody at $4^{\circ} \mathrm{C}$, rinsed with PBS, and incubated with the appropriate secondary antibody for $30 \mathrm{~min}$. Sections were counterstained with Mayer's hematoxylin, dehydrated, cleared in xylene, and mounted in Permount. Optimal antibody concentrations were determined by serial dilution in all cases. As a negative control, normal IgG was used as the primary antibody at the same dilution.

Semi-quantitative assessment and scoring. ATAD2 and $\mathrm{Hh}$ pathway gene expression levels were scored semiquantitatively according to the percentage of positively stained cells combined with the staining intensity. Samples were considered positive for ATAD2 and the Hh pathway genes if the nucleus or cytoplasm of the sample cells were positively stained. The positivity was defined as: $0,0 \% ; 1,1-10 \% ; 2,11-50 \% ; 3$, 

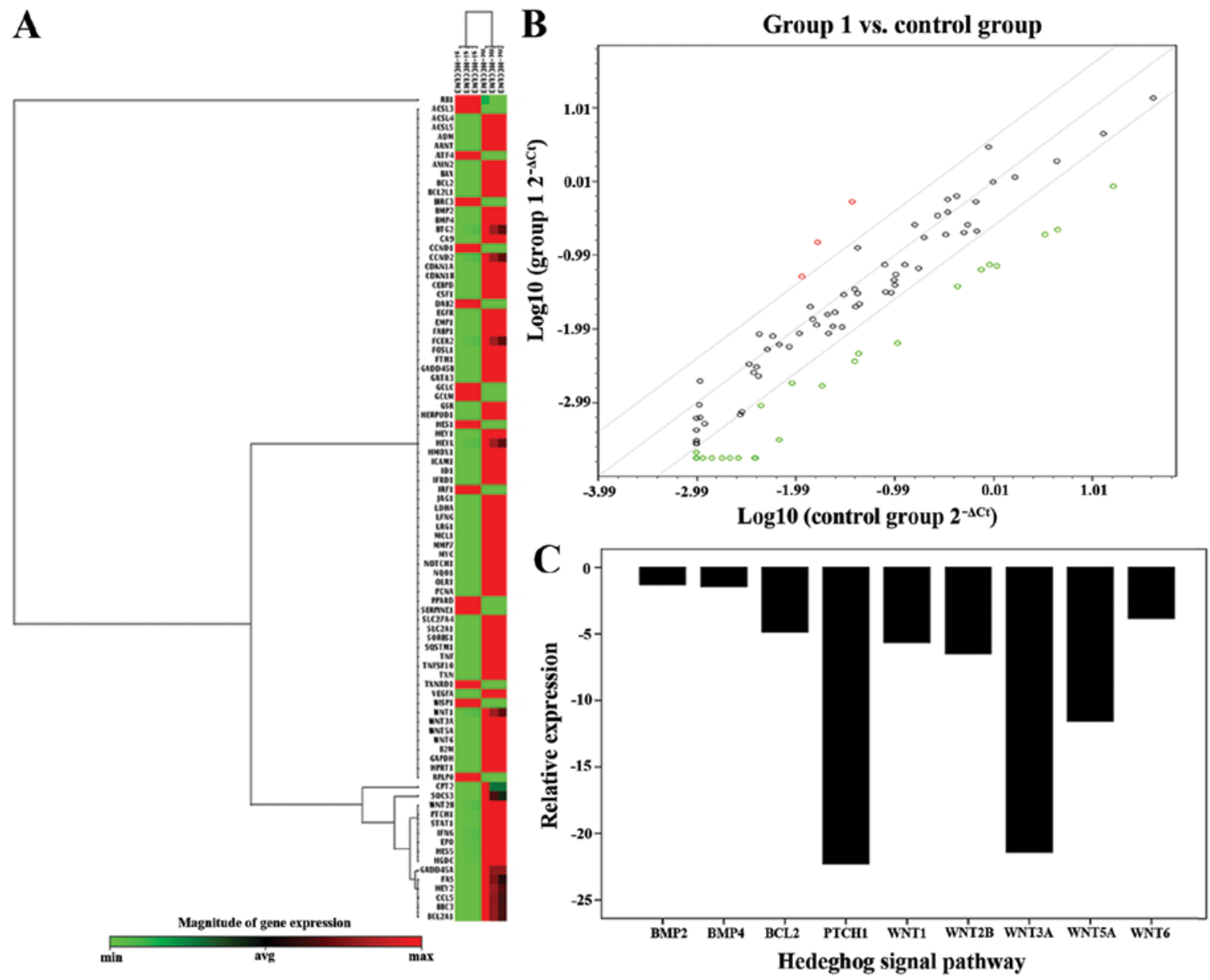

Figure 1. The image of Profiler ${ }^{\mathrm{TM}}$ PCR Array of Human Signal Transduction PathwayFinder PCR Array. (A) Clustering tree used to represent the differentially expressed genes between the normal control groups and ATAD2-RNAi-lentivirus groups. (B) Scatter plot comparison shows the changes of every gene in the two groups. The middle line indicate there was no difference in gene expression. Green scatter represent lower expression, and red represents high expression. Boundary $(-2,2)$. (C) After transfection with ATAD2-siRNA, the relationship of the mRNA expression of ATAD2 and Ptch1 was investigated, and Hh pathway was detected.

$51-80 \%$; and $4,>80 \%$. The staining intensity was scored as 0 , no staining; 1 , weakly stained; 2 , moderately stained; and 3 , strongly stained. Both the percent of positivity and the staining intensity were assessed by two investigators in a blinded manner. The ATAD2 and Hh pathway gene expression scores were calculated from the value of positivity score $\mathrm{x}$ the staining intensity score. This value thus ranged from 0 to 12 , and the tumors were classified into the following: negative (-), score 0 ; lower expression (1+), score 1-4; moderate expression (2+), score 5-8; and strong expression (3+), score 9-12. The immunohistochemical ATAD2 and Hh pathway gene staining was grouped into two categories: low expression (0/1+) and high expression $(2+/ 3+)$.

Construction and identification of the RNAi lentivirus vector. The HCCLM3 and Huh7 cells were plated and cultured in 24 -well plates until cell fusion reached $60 \%$. Next, the appropriate amounts of lentivirus were added to the cells according

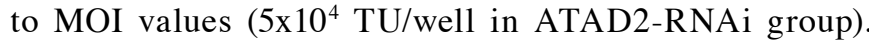
Twelve hours later, the medium was removed and replaced with fresh culture medium. Three days later, the GFP gene expression was observed under a fluorescence microscope. The effect of the RNAi-lentivirus on the expression of
ATAD2 gene was assessed by determination of the mRNA and protein levels of ATAD2 in the Huh7 and HCCLM3 cells after infection with lentivirus for 4-7 days; real-time polymerase chain reaction (PCR) and western immunoblot analyses were used for these assessments. The liver cancer cell lines Huh7, HCCLM3 were obtained from the Shanghai Cell Bank (Shanghai, China). Huh7, HCCLM3 cells were grown in DMEM (Invitrogen). All media were supplemented with $10 \%$ fetal calf serum (Invitrogen) and $100 \mathrm{IU} / \mathrm{ml}$ penicillin (Sigma, St. Louis, MO).

Cell apoptosis and cell cycle analysis. Huh7 and HCCLM3 cells in 6-well plates were transfected with ATAD2RNAi-lentivirus or control. Cells seeded at a density of $5 \times 10^{5}$ per well were trypsinized, and collected and stained using the FITC Annexin V Apoptosis Detection Kit (Keygene, China). Cell cycle analysis was performed after staining with propidium iodide (Keygene, China). Both apoptosis and cell cycle distribution were quantified using a flow cytometer.

Cell invasion and migration assay. Huh7 and HCCLM3 cells were transfected with ATAD2-RNAi-lentivirus. Cells were then seeded onto a synthetic basement membrane present 
in the inset of a 24-well culture plate. In the invasion assay, polycarbonate filters coated with $50 \mu$ l Matrigel (1:9, BD Bioscience) were placed in a Transwell chamber (Costar). In the migration assay, no Matrigel was placed in the chambers. Fetal bovine serum was added to the lower chamber as a chemoattractant. Cells were then incubated at $37^{\circ} \mathrm{C}$ and allowed to invade through the Matrigel barrier for several hours. After incubation, filters were fixed and stained with $0.1 \%$ crystal violet solution. Non-invading cells were removed using a cotton swab, and invading cells on the underside of the filter were counted with an inverted microscope.

Tumorigenicity experiments in nude mice. Forty male nude mice weighing 18 to $20 \mathrm{~g}$, provided by Shanghai Laboratory Animal Center (Chinese Academy of Science, China), were bred under aseptic conditions; the animals were housed in an area with a constant humidity of $60-70 \%$ and a room temperature of $18-20^{\circ} \mathrm{C}$. Animal maintenance, husbandry and experimental procedures were performed in accordance with the rules of China Medical University for the Use of Experimental Animals and approved by the Medical Animal Care and Use Committee of China Medical University (Shenyang, China). All of the mice were separated into two groups as described above: normal control, and ATAD2-RNAi groups. Lentivirus transfected cell groups were administered a subcutaneous injection $\left(0.1 \mathrm{ml}\right.$ of a solution containing $1 \times 10^{4}$ cells $/ \mathrm{ml})$. The mice were examined every 5 days and were sacrificed 28 days after the initial subcutaneous injection. The tumors were resected and weighed.

Pharmacological regulation of Hh signaling. The HCCLM3 line was treated with pharmacological Hh inhibitors, cyclopamine, or KAAD-cyclopamine dissolved in sterile DMSO as per the instructions of the manufacturer. For all experiments, Cyc- and KAAD-treated groups were compared to blankcontrol groups.

Statistical analyses. SPSS 19.0 for Windows was used for all analyses. The $\chi^{2}$ test was used to evaluate associations between different gene expression and clinicopathologic parameters. A Cox repression model was performed for the univariate and multivariate analysis of prognostic variables. A P-value of $<0.05$ was defined as significant.

\section{Results}

Hh pathway was detected in Human Signal Transduction PathwayFinder PCR array. To find the downstream related pathway of ATAD2, which could influence the growth of HCC, we set the two groups as normal control, and ATAD2-RNAi groups in Human Signal Transduction PathwayFinder PCR Array. The result showed that the mRNA expression of Ptch1 was significantly reduced in ATAD2-RNAi groups, compared with normal control groups (Fig. 1). Therefore, Hh pathway was detected in our study, and we speculated that ATAD2 might regulate the Hh pathway in HCC.

Altered mRNA levels of ATAD2 and the genes in Hh pathway in tissues. Mean levels of mRNA expression of ATAD2 and the genes in Hh pathway was determined by RT-PCR. Of the
80 patients, ATAD2 mRNA expression showed a 2-fold higher level in liver cancer tissue specimens, compared with normal tissues. Mean expression value of ATAD2 mRNA (4.34 \pm 1.31 and $1.35 \pm 0.79$, mean $\pm \mathrm{SD}$, respectively, $\mathrm{P}<0.05)$. The Ptch1 mRNA expression in primary HCCs tissues showed 1.5-fold, compaired with their paired non-tumorous livers. Mean expression value of Ptch1 mRNA $(3.15 \pm 1.57$ and $1.45 \pm 0.79$, mean $\pm \mathrm{SD}$, respectively, $\mathrm{P}<0.05)$. The SMO mRNA expression in primary HCCs tissues was 1-fold, compaired with their paired non-tumorous livers. Mean expression value of SMO mRNA $(2.15 \pm 0.63$ and $1.13 \pm 0.42$, mean \pm SD, respectively, $\mathrm{P}<0.05)$. The Gli2 mRNA expression in primary HCCs tissues was 6.5-fold, compaired with their paired non-tumorous livers. Mean expression value of Gli2 mRNA was $(6.75 \pm 2.54$ and $1.5 \pm 0.73$, mean $\pm \mathrm{SD}$, respectively, $\mathrm{P}<0.05$ ).

$I H C$. ATAD2 and the protein of the key genes in Hh pathway were evaluated by IHC in the $80 \mathrm{HCC}$ specimens. ATAD2 was overexpressed (2+ to $3+)$ in $67.5 \%$ tumor samples (54/80) and low-expressed (1+) in the other 11 cases $(27.5 \%)$. Ptch1 was overexpressed $(2+$ to $3+)$ in $55 \%$ of the tumor samples $(44 / 80)$ and low-expressed (1+) in the other 11 cases $(45 \%)$. SMO was overexpressed $(2+$ to $3+)$ in $52.5 \%$ of the tumor samples $(42 / 80)$ and low-expressed (1+) in the other 11 cases (47.5\%). Gli2 was overexpressed $(2+$ to $3+)$ in $72.5 \%$ of the tumor samples $(58 / 80)$ and low-expressed (1+) in the other 11 cases $(27.5 \%)$. The specimen location of ATAD2 was in the cytoplasm and cell nucleus. Ptch1 was in the cytoplasm and membrane, SOM and Glil located in the nucleus and cytoplasm. The Hh pathway genes were highly expressed in the same sites as the ATAD2 high expression, such as PTCH1, SMO, Gli2. There was a significant association between the immune intensity of ATAD2 and the protein of the key genes in Hh pathway in the same specimens (Fig. 1).

Clinical significance of ATAD2 protein expression in HCC. To evaluate the clinical significance of expression of ATAD2 and Hh pathway genes in HCC samples, we detected their protein expression by IHC, and found patients with high ATAD2 protein expression had metastases $(\mathrm{P}<0.05)$, and patients with high Ptch1 protein expression correlated with late tumor stage $(\mathrm{P}<0.05)$, and patients with high $\mathrm{Gli} 2$ protein expression had metastasis $(\mathrm{P}<0.05)$ (Table I). Patients with high SMO protein expression tended to correlate with the tumor size.

The multivariate analysis demonstrated that ATAD2 status, Ptch 1 status, Gli 2 status, the tumor size, and metastasis were significant prognostic factors for HCC patients (Table II).

Validation of ATAD2-RNAi-lentivirus and $m R N A$ and protein expression of the genes in Hh pathway in cell lines. The studies showed that ATAD2-RNAi transfection could significantly reduce the level of ATAD2 mRNA ( $<<0.05$, Fig. 2A and $\mathrm{B})$ and protein $(\mathrm{P}<0.05$, Fig. $2 \mathrm{C})$ in the ATAD2-RNAi-lentivirus group in Huh 7 cells and HCCLM3 cells, compared with the control groups. Moreover, mRNA and protein expression of the genes in Hh pathway consistently changed following the downregulation of ATAD2. The protein level in western blot analysis showed that the ratios between ATAD2 and the 
Table I. Association between ATAD2 expression and conventional clinicopathological parameters in 80 patients with HCC.

\begin{tabular}{|c|c|c|c|c|c|}
\hline Characteristics & $\mathrm{n}=80$ & $\begin{array}{c}\text { ATAD2 } \\
\text { positive }(\%)\end{array}$ & $\begin{array}{c}\text { PTCH1 } \\
\text { positive }(\%)\end{array}$ & $\begin{array}{c}\text { SMO } \\
\text { positive }(\%)\end{array}$ & $\begin{array}{c}\text { Gli2 } \\
\text { positive }(\%)\end{array}$ \\
\hline \multicolumn{6}{|l|}{ Age (years) } \\
\hline$\geq 50$ & 34 & $23(67.6)$ & $16(47.1)$ & $18(52.9)$ & $25(73.5)$ \\
\hline$<50$ & 46 & 31 (67.3) & $28(60.9)$ & $24(52.2)$ & $33(71.7)$ \\
\hline P-value & & 0.342 & 0.22 & 0.241 & 0.859 \\
\hline \multicolumn{6}{|l|}{ Gender } \\
\hline Male & 58 & $39(67.2)$ & $32(55.2)$ & $30(51.7)$ & $41 \quad(70.7)$ \\
\hline Female & 22 & $15(68.2)$ & $12(54.5)$ & $12(54.5)$ & $17(77.3)$ \\
\hline P-value & & 0.936 & 0.96 & 0.821 & 0.556 \\
\hline \multicolumn{6}{|l|}{ Tumor size } \\
\hline$\geq 5 \mathrm{~cm}$ & 56 & $39(69.6)$ & $29(51.8)$ & $32(57.1)$ & $42(75.0)$ \\
\hline$<5 \mathrm{~cm}$ & 24 & $15(62.5)$ & $15(62.5)$ & $10(41.7)$ & $16(66.7)$ \\
\hline P-value & & 0.532 & 0.544 & 0.131 & 0.251 \\
\hline \multicolumn{6}{|l|}{ Metastasis } \\
\hline Yes & 55 & $41(74.5)$ & $34(61.8)$ & $31(56.4)$ & $45(81.8)$ \\
\hline No & 25 & $13(52.0)$ & $10(40.0)$ & $11(44.0)$ & $13(52.0)$ \\
\hline P-value & & $0.046^{\mathrm{a}}$ & 0.069 & 0.305 & $0.006^{\mathrm{a}}$ \\
\hline \multicolumn{6}{|l|}{ HBsAg status } \\
\hline Positive & 44 & $29(65.9)$ & $25(56.8)$ & $22(50.0)$ & $31(70.5)$ \\
\hline Negative & 36 & $25(69.4)$ & $19(25.0)$ & $20(55.6)$ & $27(75.0)$ \\
\hline P-value & & 0.737 & 0.91 & 0.463 & 0.412 \\
\hline \multicolumn{6}{|l|}{ Differentiation } \\
\hline $\mathrm{WD}^{\mathrm{a}}$ & 25 & $17(68.0)$ & $18(72.0)$ & $12(48.0)$ & $22(88.0)$ \\
\hline $\mathrm{MD}^{\mathrm{a}}$ & 39 & $28(71.8)$ & $22(56.4)$ & $22(56.4)$ & $29(74.4)$ \\
\hline $\mathrm{PD}^{\mathrm{a}}$ & 16 & $9(56.3)$ & $4(25.0)$ & $8(50.0)$ & 7 (43.8) \\
\hline P-value & & 0.534 & 0.073 & 0.648 & 0.12 \\
\hline \multicolumn{6}{|l|}{ Cirrhosis } \\
\hline Yes & 57 & 38 (66.7) & $32(56.1)$ & $32(56.1)$ & $40(70.2)$ \\
\hline No & 23 & $16(69.6)$ & $12(52.2)$ & $12(52.2)$ & $18(78.3)$ \\
\hline $\mathrm{P}$-value & & 0.802 & 0.747 & 0.747 & 0.464 \\
\hline \multicolumn{6}{|l|}{ Serum AFP } \\
\hline$<200 \mathrm{ng} / \mathrm{dl}$ & 33 & $23(69.7)$ & $19(57.6)$ & $16(48.5)$ & $22(66.7)$ \\
\hline$\geq 200 \mathrm{ng} / \mathrm{dl}$ & 47 & $31(65.9)$ & $25(53.2)$ & $26(55.3)$ & $36(76.6)$ \\
\hline P-value & & 0.725 & 0.698 & 0.547 & 0.328 \\
\hline \multicolumn{6}{|l|}{ Tumor stage } \\
\hline $\mathrm{I}+\mathrm{II}$ & 31 & $20(64.5)$ & 12 (38.7) & $17(54.8)$ & $19(61.3)$ \\
\hline III+IV & 49 & $34(69.4)$ & $32(65.3)$ & $25(51.0)$ & 38 (77.6) \\
\hline P-value & & 0.65 & $0.02^{\mathrm{a}}$ & 0.749 & 0.117 \\
\hline
\end{tabular}

andicate statistically significant difference $(\mathrm{P}<0.05)$. WD, well differentiated; MD, moderately differentiated; PD, poorly differentiated.

key gene protein in the ATAD2-RNAi-lentivirus groups and normal control groups. The ATAD2, Ptch 1, SMO, Gli2 protein in ATAD2-RNAi were 1.5, 1.0, 1.25 and 1.25-fold, respectively, compared to normal control groups $(\mathrm{P}<0.05$, Fig. 2). This result suggests that the mRNA and protein level of Hh pathway are all reduced, after the transfection of ATAD2-RNAi lentivirus vector.
Depletion of ATAD2 influences the cell apoptosis and the cell cycle. We explored further the ATAD2 functional significance in HCC cells. To test whether expression of ATAD2 affects apoptosis in HCC cells, we performed an apoptosis assay by FACS analysis. Transfection of ATAD2-RNAi-lentivirus resulted in a significant increase in the percentage of apoptotic cells, compared with the normal control, in HCCLM3 
Table II. Univariate and multivariate analyses of individual parameters for correlations with overall survival rate: Cox proportional hazards model.

\begin{tabular}{|c|c|c|c|c|c|c|}
\hline \multirow[b]{2}{*}{ Variables } & \multicolumn{3}{|c|}{ Univariate } & \multicolumn{3}{|c|}{ Multivariate } \\
\hline & HR & CI $(95 \%)$ & P-value & HR & CI $(95 \%)$ & P-value \\
\hline ATAD2 & 1.787 & $1.150-2.684$ & $0.004^{\mathrm{a}}$ & 1.98 & $1.444-2.816$ & $0.002^{\mathrm{a}}$ \\
\hline Ptch 1 & 1.565 & $1.027-2.263$ & $0.023^{\mathrm{a}}$ & 1.833 & $1.164-2.763$ & $0.004^{\mathrm{a}}$ \\
\hline Gli 2 & 1.854 & $1.245-2.764$ & $0.002^{\mathrm{a}}$ & 1.876 & $1.274-2.773$ & $0.002^{\mathrm{a}}$ \\
\hline Gender & 1.174 & $0.601-1.583$ & 0.921 & & & \\
\hline Tumor stage & 1.126 & $0.920-1.367$ & 0.255 & & & \\
\hline Tumor differentiation & 1.029 & $0.784-1.347$ & 0.843 & & & \\
\hline Metastasis & 1.826 & $1.232-2.678$ & $0.003^{\mathrm{a}}$ & 1.842 & $1.221-2.718$ & $0.003^{\mathrm{a}}$ \\
\hline Tumor size & 1.573 & $1.059-2.336$ & $0.025^{\mathrm{a}}$ & 1.917 & $1.278-2.856$ & $0.002^{\mathrm{a}}$ \\
\hline Liver cirrhosis & 1.37 & $0.934-2.010$ & 0.107 & & & \\
\hline
\end{tabular}

HR, hazard ratio; $\mathrm{CI}$, confidence interval. ${ }^{\mathrm{a}} \mathrm{P}<0.05$ statistically significant difference.
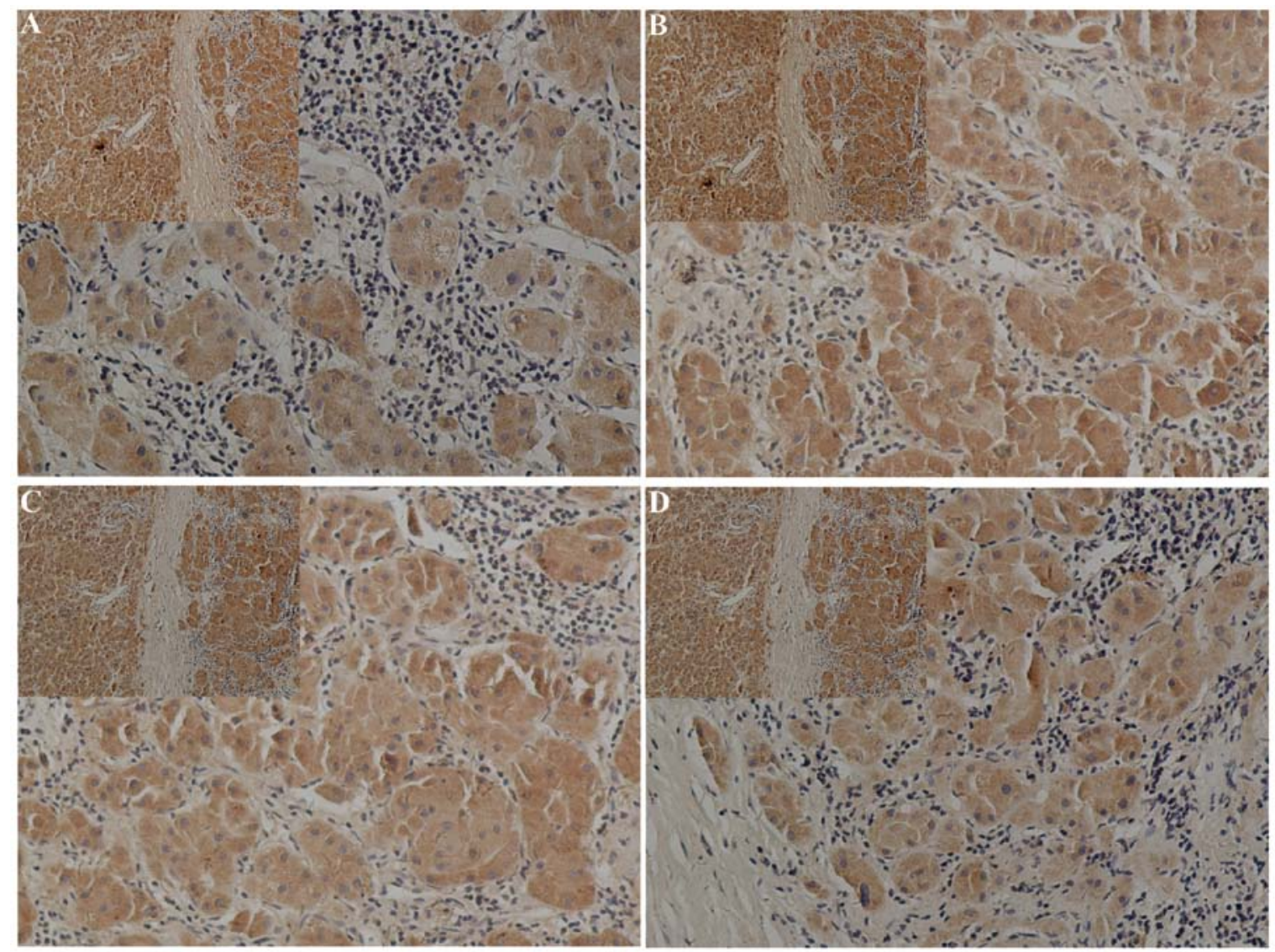

Figure 2. IHC staining of ATAD2 and Ptch 1, SMO, Gli 2 of Hh pathway in the same location of HCC tissues. Hepatic cancer tissue stained with (A) ATAD2, (B) Ptch1, (C) SMO and (D) Gli 2. Positive immunohistochemical reaction is showed as brown staining.

cells (Fig. 3A). To test whether expression of ATAD2 affects the cell cycle in HCC cells, we performed FACS cell cycle distribution analysis after $48 \mathrm{~h}$ transfection of the ATAD2-RNAi-lentivirus in HCCLM3 cells, suggesting that knockdown ATAD2 induced cell cycle arrest (Fig. 3B).

Depletion of ATAD2 influences the invasive and migratory capacity of liver cancer cells. Cell invasion and migration assays demonstrated that the Huh7 and HCCLM3 liver cancer cell lines transfected with ATAD2-RNAi-lentivirus displayed more attenuated invasive and migratory capacities than those of the normal controls (Fig. 4). The depletion of ATAD2 in Huh7 (control vs. ATAD2-RNAi: $60 \pm 11$ vs. $28 \pm 5, \mathrm{P}<0.001$ ) and HCCLM3 (control vs. ATAD2-RNAi: $72 \pm 13$ vs. $29 \pm 7$, $\mathrm{P}<0.001)$ cells led to a significant reduction in migratory cells (Fig. 4A). The invasion ability of Huh7 (control vs. ATAD2- 

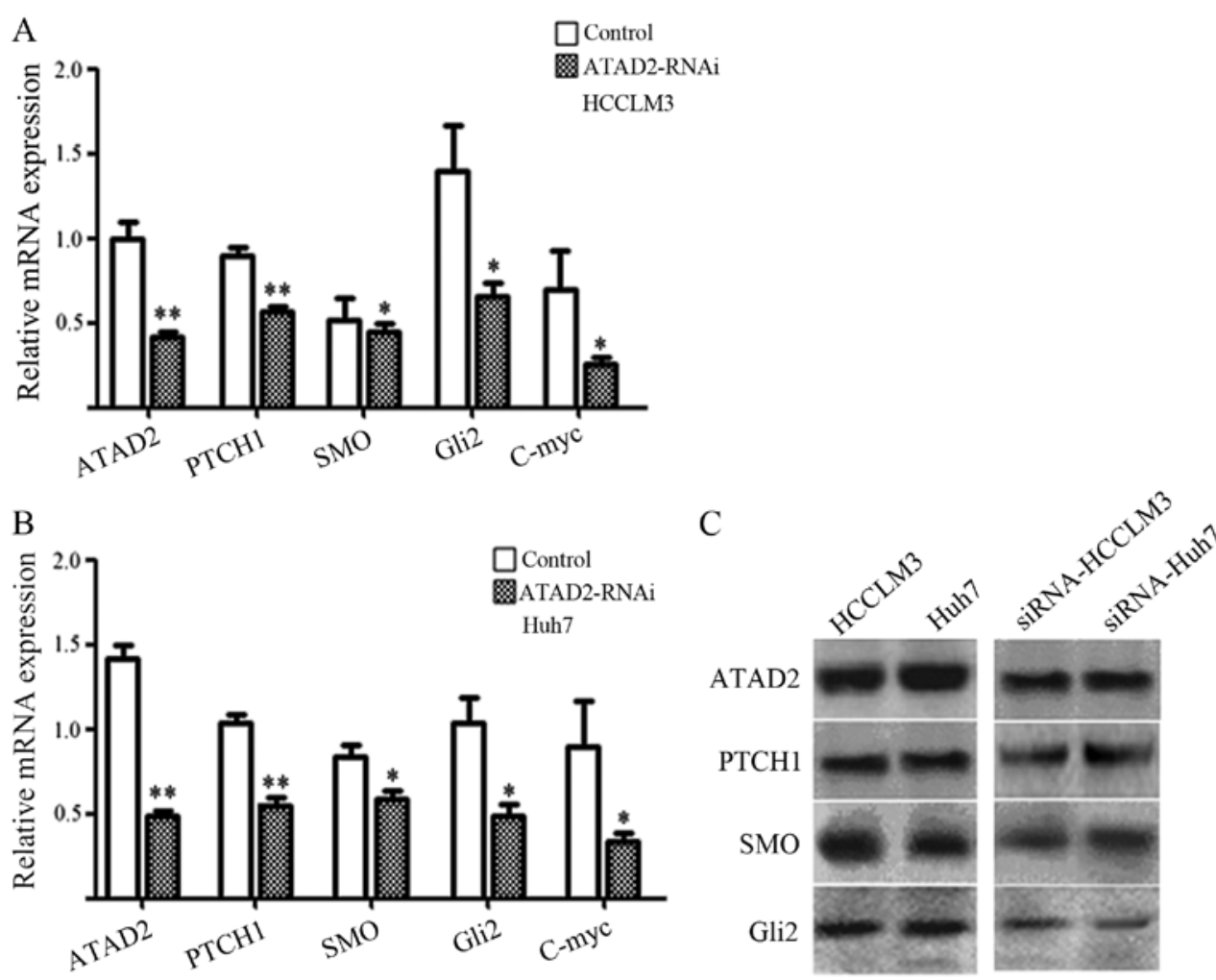

Figure 3. After transfection with ATAD2-RNAi-lentivirus, the normal HCCLM3 and Huh 7 cells showed relatively higher levels of ATAD2 and Ptch 1, SMO and Gli 2 mRNA and protein expression than the transfected groups. ${ }^{*} \mathrm{P}<0.05,{ }^{* *} \mathrm{P}<0.01$.

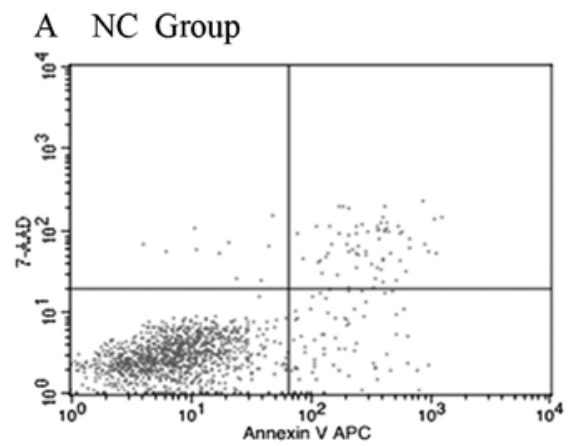

\section{B NC Group}
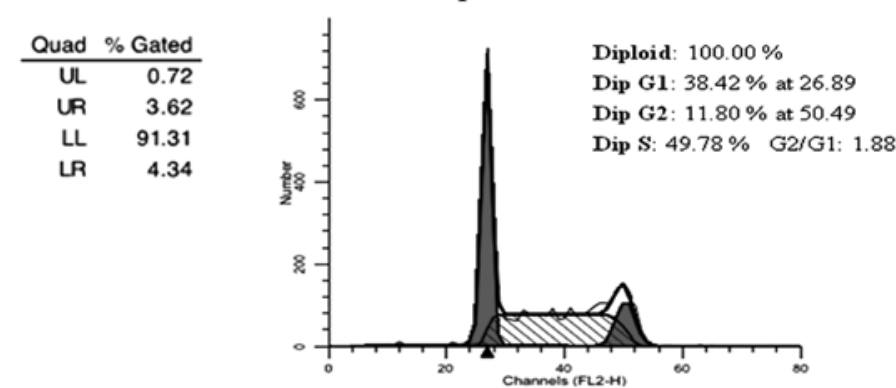

ATAD2-RNAi Group

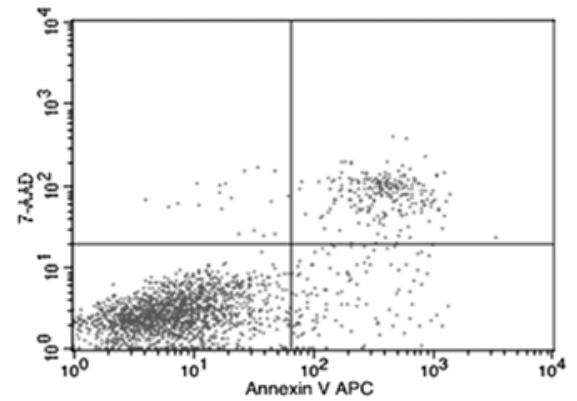

ATAD2-RNAi Group

\begin{tabular}{cr} 
Quad & $\%$ Gated \\
\hline UL & 0.81 \\
UR & 10.35 \\
LL & 83.69 \\
UR & 5.15
\end{tabular}

\begin{tabular}{|c|c|c|c|c|c|}
\hline Group & UL(\%) & UR(\%) & LL(\%) & LR(\%) & Apoptosis(\%) \\
\hline NC & 0.72 & 3.62 & 91.31 & 4.34 & 7.96 \\
\hline ATAD2-RNAi & 0.81 & 10.35 & 83.69 & 5.15 & 15.50 \\
\hline
\end{tabular}

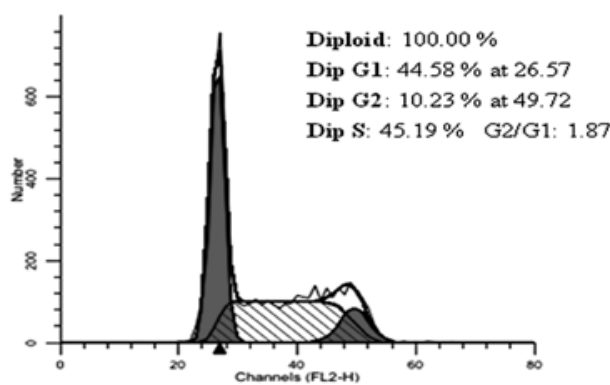

Figure 4. Depletion of ATAD2 induces apoptosis and cell cycle arrest in HCCLM3 cells. FACS analysis of ATAD2-RNAi-lentivirus or normal control cells in $48 \mathrm{~h}$ transfection. Depletion of ATAD2 significantly increased apoptosis, compared with normal control cells in HCCLM3 cells (A). Depletion of ATAD2 significantly affected the cell cycle progression in HCCLM3 cells. (B) After transfection, G1 phase arrest was detected in HCCLM3 cells, compared with normal control HCCLM3 cells. ${ }^{*} \mathrm{P}<0.05$. 

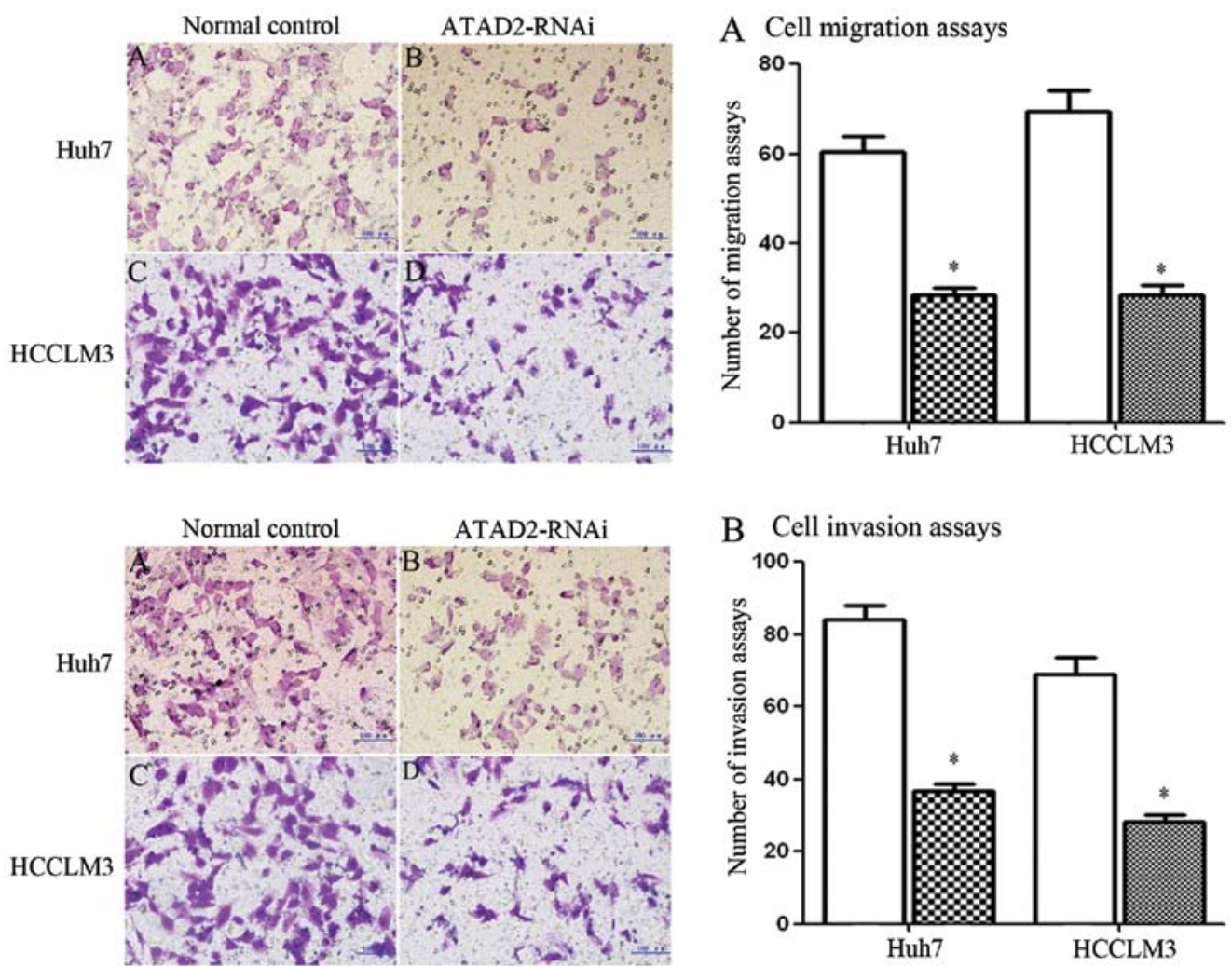

Figure 5. Transwell assays of Huh7 and HCCLM3 cells transfected with control and ATAD2-RNAi-lentivirus. (A) ATAD2 depletion had a measurable inhibitory effect on cell invasion in both cell lines. The number of invading cells were counted, and a significant difference was observed (*P<0.05). (B) The number of migrating cells were counted, and a significant difference was observed ( $\mathrm{P}<0.05)$.
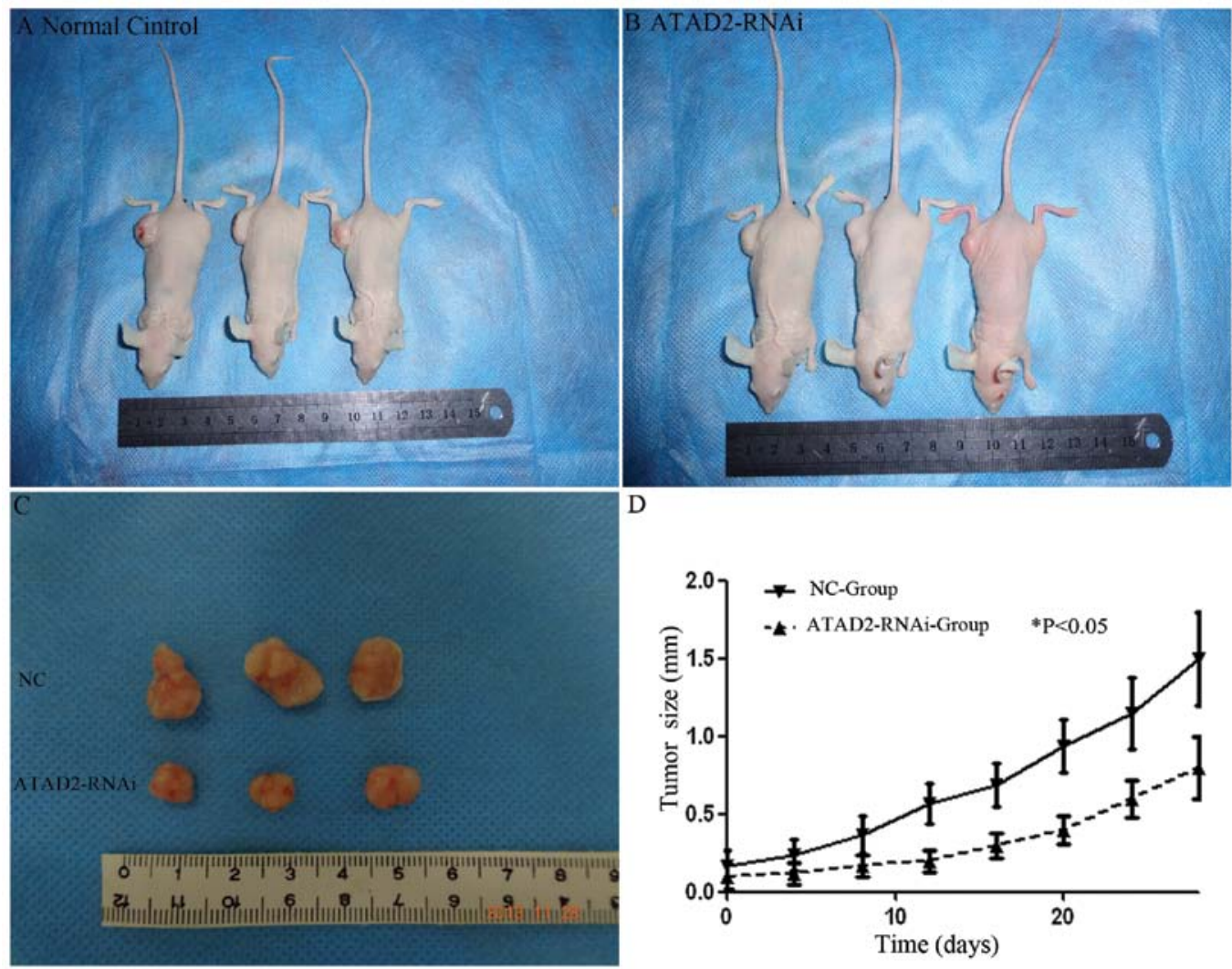

D

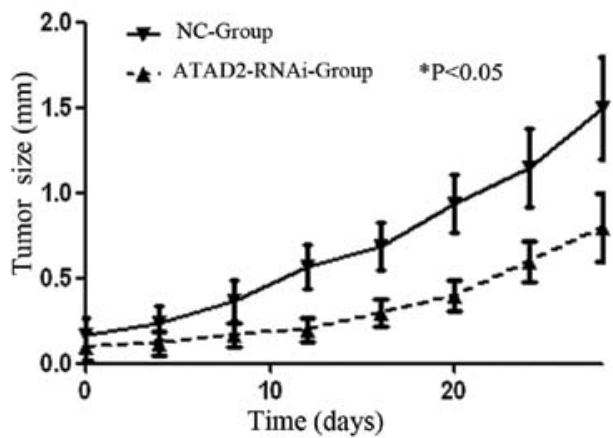

Figure 6. Inhibition of Huh 7 cell tumorigenicity in vivo after silencing of ATAD2. (A-C) ATAD2-RNAi-lentivirus-treated xenograft tumors were much smaller than normal control-treated tumors. (D) Tumor growth curves revealed that xenograft tumor growth in nude mice was significantly slower in ATAD2-RNAi-lentivirus-treated nude tumors (dashed line) than normal control treated tumors (solid line). ${ }^{*} \mathrm{P}<0.05$. 


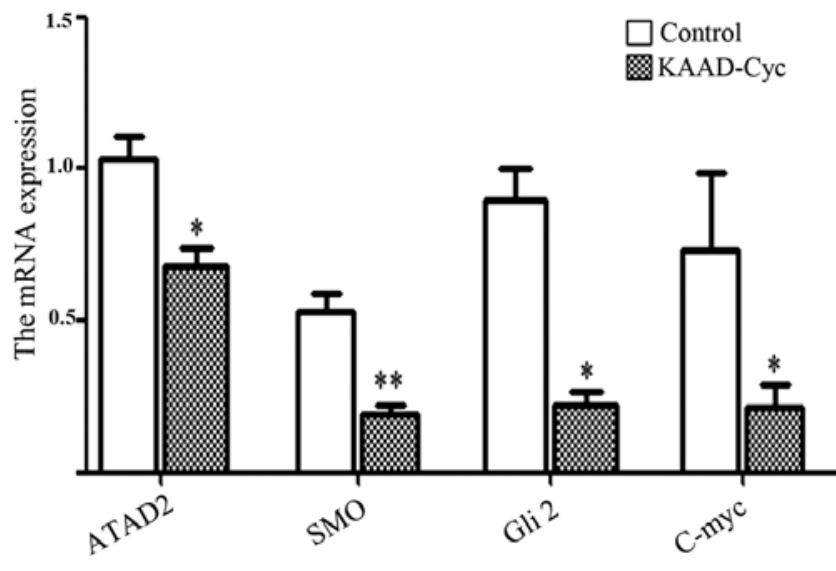

Figure 7. Quantitative RT-PCR analysis of HCCLM3 mRNA expression of ATAD2, SMO, Gli 2 and c-myc following a 5-day treatment with 1,000 nM KAAD-Cyc. Results were normalized to the normal control-treated groups; ${ }^{*} \mathrm{P}<0.05 ;{ }^{* *} \mathrm{P}<0.01$.

RNAi: $85 \pm 11$ vs. $37 \pm 5, \mathrm{P}<0.01$ ) and HCCLM3 (control vs. ATAD2 siRNA: $68 \pm 12$ vs. $27 \pm 6, \mathrm{P}<0.001)$ cells was also significantly reduced (Fig. 4B).

ATAD2-RNAi influences the cell cycle and inhibits tumor growth in nude mice. To test whether knockdown of ATAD2 could suppress the tumorigenicity of HCC cells in vivo, a xenograft model in nude mice was used. Twenty-eight days after the mice were injected with Huh7 cells and ATAD2RNAi-lentivirus transfected Huh7 cells, the weights of the tumors in the control, ATAD2-RNAi groups were $0.56 \pm 0.11$ and $0.37 \pm 0.09 \mathrm{~g}$, respectively. Thus, treatment with ATAD2RNAi inhibited the growth of tumors as compared with the control groups $(\mathrm{P}<0.05$, Fig. 6$)$.

ATAD2 may mediate MYC gene to regulate the Hh pathway. Some previous research showed that ATAD2 could cooperate with MYC in activating transcription, and ATAD2 is limited and required for the efficient transcriptional activation of a subset of MYC target genes, and Myc-interacting Zinc finger protein 1 (Mizl) as a Smo and Gli2 binding protein that positively also could regulate the Hh signaling pathway. Thus, we speculated that ATAD2 may mediate C-Myc to regulate the Hh pathway.

To investigate this hypothesis, we detected the MYC mRNA expression after transfection with ATAD2-RNAi-lentivirus. The mRNA expression of Myc was significantly reduced in Huh7 cells and HCCLM3 cells, compared with normal control $(\mathrm{P}<0.05)$ (Fig. 1), suggesting depletion of ATAD2 could reduce the mRNA expression of Myc and the key genes in the $\mathrm{Hh}$ pathway. Moreover, to test whether the Hh pathway inhibition could regulate ATAD2 and MYC expression activity, we investigated the mRNA expression of ATAD2, SMO, Gli2 and found that Myc was reduced by all treatments with KAAD-Cyc (Fig. 7). Therefore, we speculate there may be a mutual regulation between ATAD2 and the Hh pathway.

\section{Discussion}

ATAD2 has been demonstrated as a novel candidate oncogene and possibly a therapeutic target for several types of human cancer (7-10). The Hh pathway has also been demonstrated to have a strong relationship with the progression of HCC (12-14). Based on PCR array analysis, we suggested that there may be a relationship between ATAD2 and the Hh pathway. In our study, the abnormal expression of ATAD2 and Hh pathway in HCC were studied. IHC detection showed that ATAD2 was located in the nucleus and the cytoplasm, Ptch1 was located in the cytoplasm and membrane, SOM and Gli2 were located in the nucleus and cytoplasm. Furthermore, ATAD2 and Hh pathway were both consistently highly expressed in hepatic cancer tissues, but at low levels in normal liver cells. Moreover, RT-PCR also showed the high expression of ATAD2 and the Hh pathway at mRNA level. These findings provide evidence that the upregulation of ATAD2 and Hh pathway may play an important role in HCC tumorigenesis, and we speculate there may be a pathway between ATAD2 and the Hh pathway.

Combined with the clinicopathological characteristics of all tissue samples, correlation analyses indicated that the high expression of ATAD2 in the HCC tissues was positively correlated with tumor metastasis. PTCH1 was positively correlated with tumor stage, and Gli2 was positively correlated with tumor metastasis. These results demonstrated that the upregulation of ATAD2 and Hh pathway in HCC might both play an important role in promoting malignant tumors, and we suggested that ATAD2 might regulate the Hh pathway in the metastasis and proliferation of hepatic malignant tumors. Furthermore, we found that the high expression of ATAD2 and Ptch1 and Gli2 in $\mathrm{HCC}$ was a strong and independent predictor of shortened overall survival. Similar results have been observed in other human malignancies, such as esophageal, gastric, colon and breast cancers (7-14).

To elucidate the expression of ATAD2 and Hh pathway and the function of ATAD2 in HCC, in further experiments we showed that the mRNA and protein expression of ATAD2 and Ptch1, SMO and Gli2 in HCCLM3 and Huh7 cell lines, and the mRNA and protein expression of ATAD2 and Hh pathway were statistically higher in Huh7/HCCLM3 cell lines than in the ATAD2-RNAi-lentivirus. ATAD2 was able to regulate HCC cell proliferation, migration and invasion, and depletion of ATAD2 could inhibit the tumorigenicity of Huh7 cells in nude mice. These results were in agreement with previous studies that showed that ATAD2 was closely involved in several key regulatory mechanisms to control cell proliferation or tumor metastasis.

The mechanism of the impact of ATAD2 on Hh pathway is still unknown. But previous study showed that ATAD2 has a bromodomain that could mediate histone hyperacetylation and is able to bind acetylated lysines in histones and other proteins $(19,20)$. ATAD2 bromodomain appears to play a role in supporting the proliferation of cancer cells. A series of pull-down experiments suggested that ATAD2 bromodomain mediates the binding of the protein to histone $\mathrm{H} 3 / \mathrm{H} 4$ (7). However, ATAD2 also has a typical AAA ATPase domain (7). In ATAD2, this ATPase domain, like in many other members of this family, mediates protein multimerization. An inactive ATPase domain does not multimerize and cannot be caught in pull-down assays by acetylated histone, which means AAA ATPase domain has co-activator functions of ATAD2 $(6,21)$. Above all, it has been reported that ATAD2 could cooperate with MYC in activating transcription, and ATAD2 is limited 
and required for the efficient transcriptional activation of a subset of MYC target genes (7,23).

Dysregulation of the Hedgehog (Hh) pathway has been proven as implicated in the genes of hepatic cancer. Research has demonstrated that overexpression of the Smo proto-oncogene mediates c-myc-overexpression, which plays a critical role in hepatocarcinogenesis (13). Blocking the hedgehog pathway was able to inhibit proliferation, and repressed c-Myc and cyclin D expression in a subset of HCC cell lines (13). In addition, Myc-interacting zinc finger protein 1 (Miz1) as a Smo and Gli2 binding protein that positively regulated the $\mathrm{Hh}$ signaling pathway (22-26). Mizl could influence Hh activation in a Smo-dependent manner, and depletion of Mizl blocks the nuclear translocation of Gli2 (27-29). Based on above studies, we speculated ATAD2 may mediate MYC to regulate the Hh pathway activation, inducing the expression of numerous target genes, such as Ptch1, Hip, Gli and Wnt, that regulate proliferation, and differentiation $(17,30)$.

In our present study, we detected the changes in the expression of PTCH1, SMO, Gli2 and MYC with transfection of ATAD2-RNAi-lentivirus in Huh7 and HCCLM3 cell lines. The mRNA and protein of PTCH1, SMO, Gli2 and MYC respectively decreased in Huh7 and HCCLM3 cell lines. Depletion of ATAD2 negatively regulated the Hh pathway activation. Moreover, we also detected the changes in the mRNA expression of ATAD2, SMO, and Myc in HCCLM3 cell lines treated with 1,000 nM KAAD-Cyc. The results indicated that the mRNA expression of ATAD2, SMO, MYC were all decreased after being treated with 1,000 nM KAAD-Cyc, compared with the control groups. Therefore, we speculate that upregulated ATAD2 was limited and required for MYC to regulated Hh pathway to influence the proliferation and metastasis of hepatic cancer, and depletion of ATAD2 was able to inhibit the activation of the Hh pathway, MYC genes and the growth of cancer. Moreover, blocking the Hh pathway also could negatively regulate the expression of ATAD2 and MYC genes. There was an interaction between ATAD2 and the Hh pathway.

In the present study, we demonstrated the ectopic upregulation of ATAD2 and Hh pathway in HCC tissues, and ATAD2 and Gli2 indicated a poor prognosis for HCC patients. We had six reasons to clarify the relationship and function of ATAD2 and the Hh pathway in HCC in a systematic, scientific and rigorous manner: i) the mRNA and protein expression ATAD2 and the Hh pathway were upregulated in HCC and hepatic cancer cell lines; ii) the negatively downregulated mRNA and protein expression of genes in the $\mathrm{Hh}$ pathway in hepatic cancer cell lines, after being treated with transfection of ATAD2-RNAi-1; iii) depletion of ATAD2 can inhibit the proliferative and invasive capacity in HCC cell lines and the growth of hepatic cancers in nude mice; iv) previous studies proved that ATAD2 could cooperate with MYC in activating transcription, and ATAD2 is required for the efficient transcriptional activation of a subset of MYC target genes; v) Myc was able to regulate SMO and Gli genes in the Hh signaling pathway; and vi) blocking the Hh pathway also could negatively regulate the expression of ATAD2 and MYC genes, after treated with 1,000 nM KAAD or Cyc. However, further studies are needed to determine the precise mechanism underlying the role of ATAD2 in HCC progression and to make ATAD2 an attractive target for future cancer therapeutics.

In summary, we found that ATAD2 and Ptch1 were both highly expressed in HCC tissues, compared with paired normal hepatic tissues. Moreover, we found that ATAD2 could affect the expression of the Hh pathway by PCR and western blot analysis in HCC cell lines, by observing the outcome before and after transfection. We speculate that ATAD2 cooperate with MYC-regulated expression of SMO, and Gli, activating the Hh pathway and inducing the active feedback of the Hh pathway.

\section{Acknowledgements}

This study was supported by the Liaoning Provincial Committee of Education, Science and Technology Research (grant 20060903 to G.W.).

\section{References}

1. But DY, Lai CL and Yuen MF: Natural history of hepatitisrelated hepatocellular carcinoma. World J Gastroenterol 14: 1652-1656, 2008.

2. Chen HW, et al: Expression of FOXJ1 in hepatocellular carcinoma: Correlation with patients' prognosis and tumor cell proliferation. Mol Carcinog 52: 647-659, 2013.

3. He H, Wu G, Li W, Cao Y and Liu Y: CIP2A is highly expressed in hepatocellular carcinoma and predicts poor prognosis. Diagn Mol Pathol 21: 143-149, 2012.

4. Block TM, Mehta AS, Fimmel CJ and Jordan R: Molecular viral oncology of hepatocellular carcinoma. Oncogene 22: 5093-5107, 2003.

5. Feng JT, Shang S and Beretta L: Proteomics for the early detection and treatment of hepatocellular carcinoma. Oncogene 25: 3810-3817, 2006.

6. Zou JX, Revenko AS, Li LB, et al: ANCCA, an estrogen-regulated AAA+ ATPase coactivator for ERalpha, is required for coregulator occupancy and chromatin modification. Proc Natl Acad Sci USA 104: 18067-18072, 2007.

7. Ciró M, Prosperini E, Quarto M, et al: ATAD2 is a novel cofactor for MYC, overexpressed and amplified in aggressive tumors. Cancer Res 69: 8491-8498, 2009.

8. Alizadeh AA, Eisen MB, Davis RE, et al: Distinct types of diffuse large B-cell lymphoma identified by gene expression profiling. Nature 403: 503-511, 2000.

9. Chen X, Cheung ST, So S, et al: Gene expression patterns in human liver cancers. Mol Biol Cell 13: 1929-1939, 2002.

10. Ma XJ, Salunga R, Tuggle JT, et al: Gene expression profiles of human breast cancer progression. Proc Natl Acad Sci USA 100: 5974-5979, 2003

11. Huang Q, Lin B, Liu H, et al: RNA-Seq analyses generate comprehensive transcriptomic landscape and reveal complex transcript patterns in hepatocellular carcinoma. PLoS One 6: e26168, 2011.

12. Cheng WT, Xu K, Tian DY, et al: Role of Hedgehog signaling pathway in proliferation and invasiveness of hepatocellular carcinoma cells. Int J Oncol 34: 829-836, 2009.

13. Sicklick JK, Li YX, Jayaraman A, et al: Dysregulation of the hedgehog pathway in human hepatocarcinogenesis. Carcinogenesis 27: 748-757, 2006.

14. Patil MA, Zhang J, Ho C, et al: Hedgehog signaling in human hepatocellular carcinoma. Cancer Biol Ther 5: 111-117, 2006.

15. Ruiz i Altaba A, Sánchez P and Dahmane N: Gli and hedgehog in cancer: tumours, embryos and stem cells. Nat Rev Cancer 2: 361-372, 2002.

16. Ingham PW and McMahon AP: Hedgehog signaling in animal development: paradigms and principles. Genes Dev 15:3059-3087, 2001.

17. Cohen MM Jr: The Hedgehog signaling network. Am J Med Genet A 123A: 5-28, 2003.

18. Wu G, Liu H,He H, Wang Y, Lu X, et al: miR-372 down-regulates the oncogene ATAD2 to influence hepatocellular carcinoma proliferation and metastasis. BMC Cancer 14: 107, 2014. 
19. Pivot-Pajot C, Caron C, Govin J, et al: Acetylation-dependent chromatin reorganization by BRDT, a testis-specific bromodomain-containing protein. Mol Cell Biol 23: 5354-5365, 2003.

20. Filippakopoulos P, Picaud S, Mangos M, et al: Histone recognition and large-scale structural analysis of the human bromodomain family. Cell 149: 214-231, 2012.

21. Caron C, Lestrat C, Marsal S, et al: Functional characterization of ATAD2 as a new cancer/testis factor and a predictor of poor prognosis in breast and lung cancers. Oncogene 29: 5171-5181, 2010.

22. Seoane J, Pouponnot C, Staller P, Schader M, Eilers M, et al: TGFbeta influences Myc, Miz-1 and Smad to control the CDK inhibitor p15INK4b. Nat Cell Biol 3: 400-408, 2001.

23. Leachman NT, Brellier F, Ferralli J, et al: ATAD2B is a phylogenetically conserved nuclear protein expressed during neuronal differentiation and tumorigenesis. Dev Growth Differ 52: 747-755, 2010

24. Lu J, Chen M, Ren XR, et al: Regulation of Hedgehog signaling by Myc-interacting zinc finger protein 1, Miz1. PLoS One 8 e63353, 2013.
25. Peukert K, Staller P, Schneider A, et al: An alternative pathway for gene regulation by Myc. EMBO J 16: 5672-5686, 1997.

26. Wanzel M, Herold S and Eilers M: Transcriptional repression by Myc. Trends Cell Biol 13: 146-150, 2003.

27. Patel JH and McMahon SB: BCL2 is a downstream effector of MIZ-1 essential for blocking c-MYC-induced apoptosis. J Biol Chem 282: 5-13, 2007.

28. Rao G, Pedone CA, Coffin CM, Holland EC and Fults DW: c-Myc enhances sonic hedgehog-induced medulloblastoma formation from nestin-expressing neural progenitors in mice. Neoplasia 5: 198-204, 2003.

29. Wang Y, Wu MC, Sham JS, et al: Prognostic significance of c-myc and AIB1 amplification in hepatocellular carcinoma. A broad survey using high-throughput tissue microarray. Cancer 95: 2346-2352, 2002.

30. Shachaf CM, Kopelman AM, Arvanitis C, et al: MYC inactivation uncovers pluripotent differentiation and tumour dormancy in hepatocellular cancer. Nature 431: 1112-1117, 2004. 